\title{
Loss of Lag Response Curvilinearity of Gait Poincare Plot Indices in Neurodegenerative Disorders
}

\author{
Chandrakar Kamath \\ Shantha Nilaya, Ananthnagar, Manipal, India \\ Email: kamath@gmail.com
}

Received 27 May 2015; accepted 13 June 2015; published 19 June 2015

Copyright (C) 2015 by author and OALib.

This work is licensed under the Creative Commons Attribution International License (CC BY). http://creativecommons.org/licenses/by/4.0/

(c) (†) Open Access

\section{Abstract}

It is reported in the literature that the temporal structure of gait variability in healthy subjects exhibits deterministic processes where not only each stride is correlated with the neighbouring strides (i.e. short-range correlations), but at least on a statistical basis, with tens and hundreds of preceding and following strides (i.e. long-range correlations). Thus, an analysis hinging on a conventional gait Poincare plot with lag one which implicitly assumes that the current stride is influenced by the immediately preceding stride will likely underestimate the role of the autocovariance function of stride intervals. This implies that a series of lagged gait Poincare plots can potentially provide more information by reflecting short-range correlations of gait variability through the behaviour of Poincare indices in health as well as disease. Hence, in this study in the context of short-term variability, we assessed a curvilinear relation between lag (1 - 6) and Poincare indices in normal subjects and patients with neurodegenerative disorders. We found that while normal subjects exhibited this curvilinearity, the patients with neurodegenerative disorders showed its loss.

\section{Keywords}

Gait Signal, Neurodegenerative Disorder, Nonlinear Dynamics, Ordinal Patterns, Symbolic Analysis

Subject Areas: Biological Engineering, Neuroscience

\section{Introduction}

Gait is a complicated process involving coordination of multiple systems within the body (e.g. central nervous, musculoskeletal, and cardiovascular systems) [1]. For a person to walk, the nervous system has to send signals to control a large number of muscles and at the same time process sensory information to monitor and refine 
movements, all while maintaining an upright stance [1]. Thus the gait variability arises from a combination of factors [2]. Gait variability, defined as the fluctuations in gait characteristics between strides, is found to be low during walking [1]. However, increased or decreased variability is found in subjects with gait abnormalities, like elderly fallers and patients with neurodegenerative disorders (e.g. Parkinson’s and Huntington's diseases). Increased variability is associated with balance impairments, central nervous system impairments (such as cognitive functioning and motor control function), while decreased variability is associated with sensory impairments. Thus, gait variability reflects walking impairments and can be readily used to assess the motor performance. Long-term structure in gait variability has been well studied and documented [3]-[5]. These studies indicate that the temporal structure of gait variability in healthy subjects shows evidence of deterministic processes where not only each stride is correlated with the neighbouring strides (i.e. short-range correlations), but at least on a statistical basis, with tens and hundreds of preceding and following strides (i.e. long-range correlations). The purpose of this study is to determine the distinguishing characteristic of short-range correlations in neighbouring gait cycles during short gait sessions of normal subjects and patients with neurodegenerative disorders. Such short gait sessions are common in clinical applications. A conventional gait Poincare plot with lag one captures only the single lag correlation in the gait time series. Thus, an analysis banking on the use of only successive stride interval duplets will likely underestimate the role of the autocovariance function of stride intervals i.e., the ability of gait to influence a train of succeeding strides. Therefore, a series of multiple-lag gait Poincare plots can potentially provide more insight by reflecting short-range correlations of gait variability through the behaviour of Poincare indices in health as well as disease. Hence, in addition to this conventional plot $\left(x_{n+1}\right.$ against $\left.x_{n}\right)$ we also used the generalized Poincare plot with different lags i.e., the $m$-lagged Poincare plot (plot of $x_{n+m}$ against $x_{n}$ ), where $m$ represented the lag. The concept of this $m$-lagged gait Poincare plot emerged from the rationale that any given stride interval could influence many of the subsequent stride intervals. We hypothesized that assessment of stride-to-stride aspects of stride interval changes at different lags would differentiate the healthy controls from those with neurodegenerative diseases. In this study, we use three databases of neurodegenerative diseases, amyotrophic lateral sclerosis (ALS), Parkinson's disease (PD), and Huntington's disease (HD), for comparison with healthy controls. ALS is a motor neuron disease, while PD and HD are associated with disorders of the basal ganglia. ALS patients display an abnormal gait with decreased walking velocity. In its most classical manifestation PD patients exhibit bradykinesia (i.e., slowed movements), hypokinesia (i.e., small amplitude movements), muscular rigidity, postural instability, and resting tremor. This implies increased stride variability in PD. On the other hand, HD patients exhibit an uncoordinated, lurching walk. As a result of these pathologies, in all the cases, the fluctuation magnitude and the stride-to-stride dynamics of gait are impaired. Capturing stride dynamics through the use of lagged Poincare plots we provide interesting insights into dynamics of gait. To the best of our knowledge, this is the first study which unravels the short-range correlations of gait variability through the behaviour of Poincare indices in health as well as disease, evaluates curvilinearity in the lag response and separates the healthy from the aforementioned neurological disordered groups.

\section{Methods and Materials}

The paper is organized as follows. Section 2.1 discusses the database which is widely used in stride analysis. Section 2.2 discusses how the pre-processing of the gait data is carried out in this work. Sections 2.3 explains the gait Poincare plot and the Poincare indices. Section 2.4 deals with $m$-lagged Poincare plot and its advantages over conventional Poincare plot. Section 2.5 deals with lag-response analysis and curvilinearity. Section 2.6 discusses statistical tests used. In Section 3 we discuss the results.

\subsection{Database}

The database used in this study is from subjects recruited in Neurology Outpatient Clinic at Massachusetts General Hospital, Boston, USA, and is contributed by Hausdorff et al. [6] [7] to public domain and can be downloaded from the physionet.org [8]. The neurodegenerative disease records in this database include stride time series from 13 ALS patients (10 males and 3 females, age mean \pm standard deviation: $55.6 \pm 12.8$ years), 15 PD patients (10 males and 5 females, age mean \pm standard deviation: $66.80 \pm 10.85$ years), $20 \mathrm{HD}$ patients (6 males and 14 females, age mean \pm standard deviation: $46.65 \pm 12.60$ years).The patients of PD were, on average, older than both other groups. The subjects with neurodegenerative disorder were selected based on their ability to walk independently for 5 minutes. It was confirmed that the patients free from other pathologies which might 
lead to lower extremity weakness only participated. Over the duration of treatment the medication usage was sustained. The database also includes records from 16 healthy control subjects ( 2 males and 14 females, age mean \pm standard deviation: $39.3 \pm 18.5$ years). These control subjects were included from general community. It is to be noted that heights and weights in the four groups were not significantly different. It was also confirmed that the healthy subjects were free from visual, respiratory, cardiovascular, or other neurological diseases. All the subjects were asked to provide written consent to the hospital and the MGH institutional Review board had provided approval for the study [6] [7].

The subjects from the four groups were instructed to walk at their normal pace up and down a $77 \mathrm{~m}$ long hallway for $5 \mathrm{~min}$. To measure the gait rhythm and the timing of the gait cycle, force sensitive insoles were place inside or under subject's shoes. These sensors produce a measure proportional to the force applied to the ground during movement. The output from the footswitches which corresponds to force signal is sampled at 300 $\mathrm{Hz}$ and digitized using an analog-to-digital converter and then stored in a recorder. The recorded data is then analyzed using a validated software that determined initial and end contact times (and also, stride and swing times) of each stride.

\subsection{Pre-Processing the Gait Data}

It is necessary to pre-process the gait data before the application of the method of analysis. The samples in the first 20 seconds of the recordings are removed to minimize the start-up effects [6]. Over the monitoring interval of 5 minutes, each time the subject reached the end of the hall-way the subject had to turn around and continue walking. The strides associated with these turning events are to be treated as outliers and should be removed from the rest of the time series. The three-sigma-rule [9], which states that $99.7 \%$ of the normally distributed probability values lie within the range of (mean $\pm 3 . S D$ ) where SD is the standard deviation, is employed to remove the outliers. This means that those samples which lie outside the range (median $\pm 3 . S D$ ) are outliers and hence, can be removed. In the removal process, median value and not mean value of the time series has been used because some outliers possessed large values and therefore, will affect the computation of the mean.

\subsection{Gait Poincare Plots and Poincare Indices}

A conventional Poincare plot is a geometrical representation of a time series into a Cartesian plane, where the values of each pair of successive elements of the time series define a point in the scatter plot [10]-[12]. In the case of gait analysis each stride interval is plotted against its predecessor in the scatter plot. This procedure provides an indication of the probability of occurrence of one interval from its predecessor and allows assessment of dynamic properties of stride interval variation. The indices of stride variability are strongly correlated with the length, width and shape of the resulting cloud of points (Poincare cloud) dispersed along the line of identity $(y=x)$ in the scatter plot. The Poincare cloud is usually characterized by its length $\left(\mathrm{SD}_{2}\right)$ along the line of identity and its width $\left(\mathrm{SD}_{1}\right)$ perpendicular to this line. The scatter plot width $\left(\mathrm{SD}_{1}\right)$ is closely related to short-term variability in stride intervals; scatter plot length $\left(\mathrm{SD}_{2}\right)$ is correlated with long-term variability parameters. The ratio $\mathrm{SD}_{1} / \mathrm{SD}_{2}$, designated by $\mathrm{SD}_{12}$, is a measure of the shape of Poincare plot [13]. The Poincare indices have been shown to be a function of the autocorrelation of the time series at different lags [10].

A Poincare plot is analyzed quantitatively by evaluating $\mathrm{SD}_{2}$ and $\mathrm{SD}_{1}$, the dispersions of points along the line $y=x$ and the line $y=-x+2 * X_{m}$, respectively, where $X_{m}$ represents the mean of the stride interval series. The intersection of these two lines is given by $\left(X_{m}, X_{m}\right)$.

\section{4. $m$-Lagged Gait Poincare Plots}

A generalized Poincare plot with different lags, also called $m$-lagged Poincare plot, is a plot of stride interval $x_{n+m}$ against stride interval $x_{n}$, where $m$ represents the lag. Lerma et al. have found that heart rate variability measurements from a series of lagged Poincare plots (multiple lag correlation) can give more particulars about the behaviour of Poincare plot than those from the conventional 1-lag plot [14]. Thakre et al. examined the theoretical demand with different lags in heart rate variability studies and showed that there was a quadratic relationship between lag and Poincare indices in normal subjects, which was lost in congestive heart failure patients [15]. It is reported in the literature that the temporal structure of gait variability in healthy subjects exhibits deterministic processes where not only each stride is correlated with the neighbouring strides (i.e. short-range 
correlations), but at least on a statistical basis, with tens and hundreds of preceding and following strides (i.e. long-range correlations). Thus, an analysis solely dependent on a conventional gait Poincare plot with lag one, which implicitly assumes that the current stride is influenced by the immediately preceding stride, is likely to undervalue the role of the autocovariance function of stride intervals. This clearly implies that using a series of $m$-lagged gait Poincare plots can potentially provide more information by reflecting short-range correlations of gait variability through the performance of Poincare indices. Hence, in this study in the context of short-term variability, we assessed a quadratic/curvilinear relation between lag $(1-6)$ and Poincare indices in normal subjects and patients with neurodegenerative disorders.

\subsection{Lag-Response Analysis and Curvilinearity}

To study the effect of lag on Poincare indices we employed $m$-lagged Poincare plots. For each plot, Poincare indices $\mathrm{SD}_{1}, \mathrm{SD}_{2}$, and $\mathrm{SD}_{12}$ were computed and those at a particular lag were averaged in each group (from healthy controls, ALS, PD, and HD groups). Analysis of lag-response involves plotting of these estimates of Poincare indices against lag, commonly called lag-responses and then trying to fit a second order polynomial curve using the least-squares method to establish a quadratic relationship. The model-fit is assessed using $\mathrm{R}^{2}$ values, $0 \leq R^{2} \leq 1.0$. The closer the value of $R^{2}$ to 1.0 the better is the fit and closer the value to 0 worse is the fit. The averaged coefficients of the quadratic terms in the second order polynomial equations serve as markers for curvilinearity. Higher values of these coefficients indicate more curvilinearity as shown by higher curvature in the lag response and lower values indicate less curvilinearity as shown by diminished curvature in the lag response.

\subsection{Statistical Analysis}

The curvilinearity of lag-response of a Poincare index $\left(\mathrm{SD}_{1}, \mathrm{SD}_{2}\right.$, or $\left.\mathrm{SD}_{12}\right)$ was assessed using quadratic polynomial regression and the model-fit was assessed using $R^{2}$ values, $0 \leq R^{2} \leq 1$ as mentioned above. For comparison between the $m$-lagged Poincare indices of healthy control and neurodegenerative disorders we used initially nonparametric Kruskal-Wallis test. For pair-wise comparisons between groups we employed nonparametric Mann-Whitney rank sum test. When the data do not meet the requirements for a parametric test (i.e. if the data are not normally distributed), as in gait data, it is advisable to employ nonparametric tests. To perform MannWhitney rank sum test, first rank all the values from low to high with no regard for which group it belongs to. If two values are same, then they both get the average of the two ranks for which they tie. The smallest among values gets rank 1 and the largest gets a rank $\mathrm{N}$. N represents the total number of values in the two groups. Next, to find the test statistic, sum the rank of one population and report the sum. If the samples are small and there are no ties, an exact $p$-value will result. If the samples are large or if there are ties, an approximate $p$-value can be computed from a Gaussian approximation. The Kruskal-Wallis testis an extension of the Mann-Whitney rank sum test that permits simultaneous testing of the multiple groups. For all statistical analysis, the significance was fixed at $p<0.05$.

\section{Results and Discussion}

In order to compare the gait patterns in healthy control and neurodegenerative disorder subjects we plotted Poincare plots at two different lags. Figure 1 shows representative gait Poincare plots with stride segment length $=500$ and lag $=1$ for subjects from healthy controls, ALS, PD, and HD groups and Figure 2 shows representative gait Poincare plots with stride segment length $=500$ and lag $=6$ for the same subjects in the same order. The first thing to observe is that the dispersion of points in a particular plot is more in Figure $2(\operatorname{lag}=6)$ than the corresponding plot in Figure 1 (lag $=1)$ for the same group. Visual analysis of the plots shows that the gait variability increases with lag in all the cases. This indicates that increased lag corresponds to increased unrelated strides. The second point to note is that at each lag the gait Poincare plots for neurodegenerative disorder cases exhibit more dispersion of points and hence higher variability compared to that of healthy control. Our hypothesis was that assessment of stride-to-stride aspects of stride interval changes at different lags would capture this behaviour and differentiate the healthy controls from those with neurodegenerative diseases. The variability in the plot reflects the performance of the locomotor system in controlling the strides. As mentioned above, $\mathrm{SD}_{1}$ and $\mathrm{SD}_{2}$, respectively serve as short-term and long-term variability measures. Many researchers have observed 


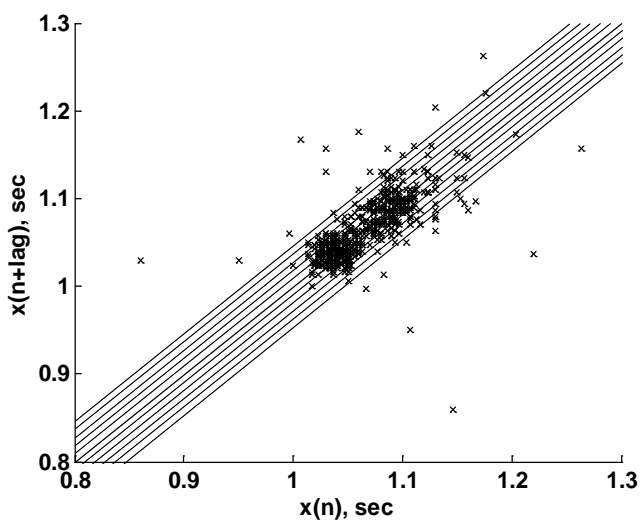

(a)

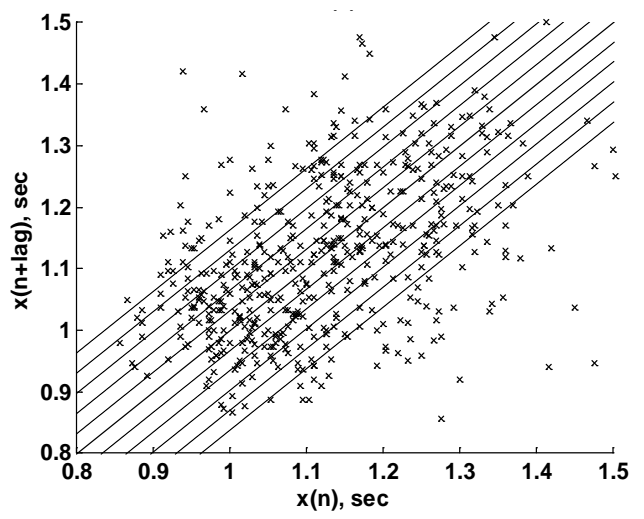

(c)

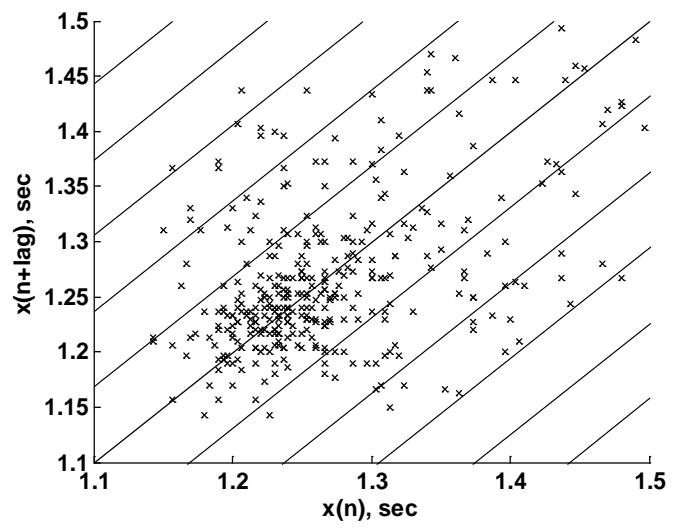

(b)

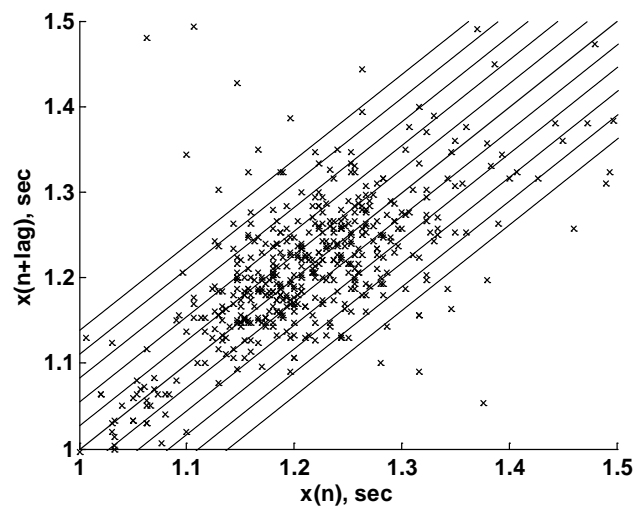

(d)

Figure 1. Poincare plots of $x(n+\operatorname{lag})$ vs. $x(n)$ for (a) control subject, (b) ALS patient, (c) Huntington's patient, and (d) Parkinson's patient at lag $=1$ and stride segment length $=500$.

that short-term recordings are equally reliable and accurate as long-term recordings. Therefore, we explored the lag-response of gait variability for stride segments with different lengths from 200 to 700 strides in steps of 50 strides. Thus, for each group (from healthy controls, ALS, PD, and HD groups) we used stride segments of 10 different lengths. For each segment, we used lags from 1 to 6 . For each lag, the Poincare indices $\left(\mathrm{SD}_{1}, \mathrm{SD}_{2}\right.$, and $\mathrm{SD}_{12}$ ) were computed. We first assessed the influence of stride segment length on the Poincare indices at lag $=1$. A summary of these indices at lag $=1$, expressed as mean \pm SD, for different stride segment lengths (200 to 700 strides in steps of 50 strides) are shown in Table 1 through Table 3. As mentioned above, Kruskal-Wallis test was employed to evaluate the statistical significance between the Poincare indices of healthy control (HC) and neurodegenerative disorders groups. The results are tabulated in last two columns of Table 1 through Table 3 . The statistical significance shows that while $\mathrm{SD}_{1}$ and $\mathrm{SD}_{2}$ can readily separate healthy control from neurodegenerative disorder subjects, $\mathrm{SD}_{12}$ cannot. This is because both $\mathrm{SD}_{1}$ and $\mathrm{SD}_{2}$ are increased simultaneously in neurodegenerative disorder subjects compared to those of healthy control subjects and the $\mathrm{SD}_{12}$ ratios tend to be nearly same. This makes $\mathrm{SD}_{12}$ statistically insignificant to discern $\mathrm{HC}$ and neurodegenerative disorders groups. Also, the statistical significance $\mathrm{SD}_{1}$ and $\mathrm{SD}_{2}$ is found to decrease with increasing segment length. To assess the difference between each of $\mathrm{SD}_{1}, \mathrm{SD}_{2}$, and $\mathrm{SD}_{12}$ for binary classification we used Mann-Whitney rank sum test and the results are shown in Table 4. The $p$-values indicate the same findings mentioned above.

Next, to assess the influence of lag on the estimates of Poincare indices $\left(\mathrm{SD}_{1}, \mathrm{SD}_{2}\right.$, and $\left.\mathrm{SD}_{12}\right)$ we employed $m$-lagged Poincare plots with lag $m$ varied from 1 to 6 . As mentioned in Section 2.4, the lag response was examined and the curvilinearity was assessed in each group, in particular, for three segment lengths: 200, 500, and 700 strides. Figure 3 through Figure 5 illustrate the effect of lag on $\mathrm{SD}_{1}, \mathrm{SD}_{2}$, and $\mathrm{SD}_{12}$ for each group. Distinct curvilinearity can be seen in the lag response of healthy controls as compared to those of neurodegenerative disorder groups at all segment lengths. The corresponding averaged coefficients of the quadratic terms for best 


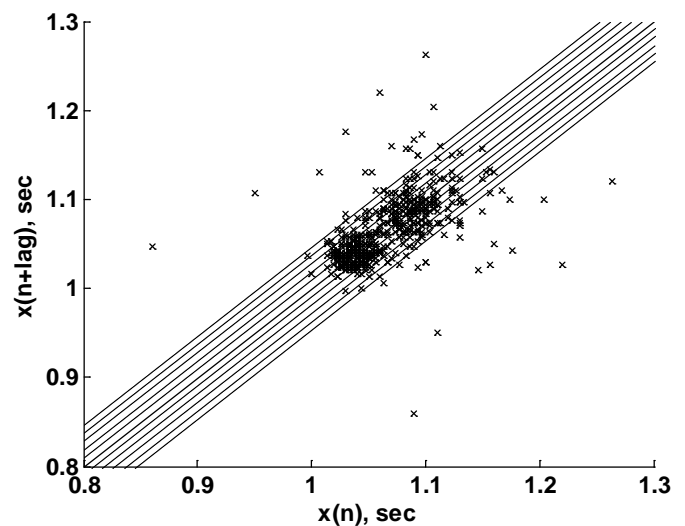

(a)

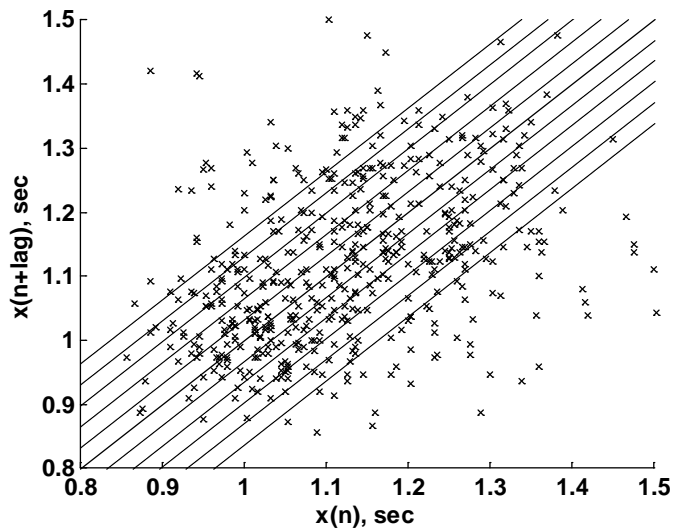

(c)

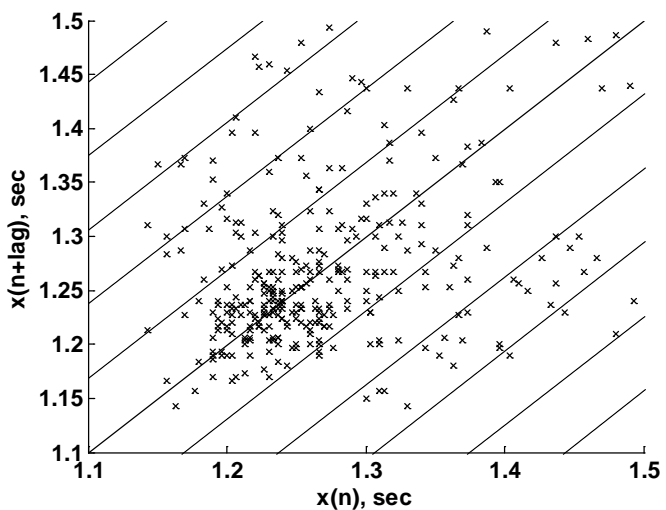

(b)

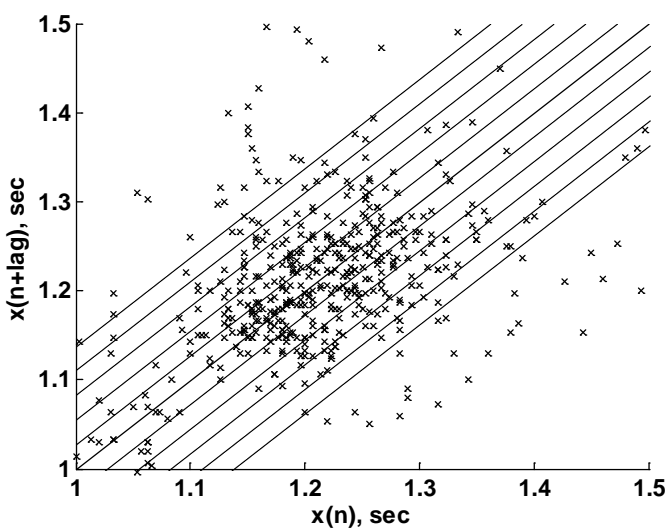

(d)

Figure 2. Poincare plots of $x(n+\operatorname{lag})$ vs. $x(n)$ for (a) control subject, (b) ALS patient, (c) Huntington's patient, and (d) Parkinson's patient at lag $=6$ and stride segment length $=500$.

Table 1. Comparison of Poincare index $\mathrm{SD}_{1}$ for different stride segment lengths at lag $=1$ in the four groups (HC, ALS, PD, and HD). All the values are expressed as mean \pm SD. Seg. Len: stride segment length, HC: healthy control, ALS: amyotrophic lateral sclerosis, PD: Parkinson’s disease, and HD: Huntington’s disease.

\begin{tabular}{ccccccc}
\hline \multirow{2}{*}{$\begin{array}{c}\text { Seg. } \\
\text { Len. }\end{array}$} & \multicolumn{2}{c}{ SD } & \multicolumn{2}{c}{ Kruskal-Wallis test } \\
\cline { 2 - 7 } & $\mathrm{HC}$ & $\mathrm{ALS}$ & $\mathrm{PD}$ & $\mathrm{HD}$ & Chi-sq & $p$-value \\
\hline 200 & $0.025 \pm 0.002$ & $0.087 \pm 0.042$ & $0.060 \pm 0.015$ & $0.076 \pm 0.018$ & 37.99 & $2.847 \times 10^{-08}$ \\
250 & $0.026 \pm 0.002$ & $0.072 \pm 0.016$ & $0.065 \pm 0.017$ & $0.080 \pm 0.179$ & 32.11 & $4.968 \times 10^{-07}$ \\
300 & $0.024 \pm 0.002$ & $0.107 \pm 0.057$ & $0.066 \pm 0.016$ & $0.095 \pm 0.032$ & 28.68 & $2.609 \times 10^{-06}$ \\
350 & $0.025 \pm 0.002$ & $0.112 \pm 0.063$ & $0.066 \pm 0.016$ & $0.093 \pm 0.028$ & 24.93 & $1.599 \times 10^{-05}$ \\
400 & $0.025 \pm 0.002$ & $0.119 \pm 0.067$ & $0.063 \pm 0.016$ & $0.098 \pm 0.033$ & 21.34 & $8.543 \times 10^{-05}$ \\
450 & $0.025 \pm 0.002$ & $0.115 \pm 0.076$ & $0.071 \pm 0.018$ & $0.097 \pm 0.034$ & 18.11 & 0.0004 \\
500 & $0.025 \pm 0.002$ & $0.114 \pm 0.063$ & $0.066 \pm 0.018$ & $0.094 \pm 0.037$ & 16.07 & 0.0011 \\
550 & $0.025 \pm 0.002$ & $0.131 \pm 0.065$ & $0.065 \pm 0.023$ & $0.096 \pm 0.036$ & 15.41 & 0.0015 \\
600 & $0.025 \pm 0.003$ & $0.124 \pm 0.058$ & $0.074 \pm 0.011$ & $0.092 \pm 0.032$ & 13.21 & 0.0042 \\
650 & $0.025 \pm 0.001$ & $0.130 \pm 0.060$ & $0.074 \pm 0.014$ & $0.100 \pm 0.032$ & 13.22 & 0.0042 \\
700 & $0.025 \pm 0.001$ & $0.123 \pm 0.066$ & $0.690 \pm 0.015$ & $0.092 \pm 0.028$ & 11.22 & 0.0106 \\
\hline
\end{tabular}


Table 2. Comparison of Poincare index $\mathrm{SD}_{2}$ for different stride segment lengths at lag $=1$ in the four groups (HC, ALS, PD, and HD). All the values are expressed as mean \pm SD. Seg. Len: stride segment length, HC: healthy control, ALS: amyotrophic lateral sclerosis, PD: Parkinson's disease, and HD: Huntington’s disease.

\begin{tabular}{|c|c|c|c|c|c|c|}
\hline \multirow{2}{*}{$\begin{array}{l}\text { Seg. } \\
\text { Len. }\end{array}$} & \multicolumn{4}{|c|}{$\mathrm{SD}_{2}$} & \multicolumn{2}{|c|}{ Kruskal-Wallis test } \\
\hline & $\mathrm{HC}$ & ALS & $\mathrm{PD}$ & HD & Chi-sq & $p$-value \\
\hline 200 & $0.053 \pm 0.007$ & $0.176 \pm 0.089$ & $0.116 \pm 0.032$ & $0.113 \pm 0.027$ & 24.72 & $1.770 \times 10^{-05}$ \\
\hline 250 & $0.049 \pm 0.005$ & $0.189 \pm 0.094$ & $0.118 \pm 0.033$ & $0.132 \pm 0.039$ & 24.05 & $2.436 \times 10^{-05}$ \\
\hline 300 & $0.056 \pm 0.002$ & $0.241 \pm 0.060$ & $0.136 \pm 0.028$ & $0.137 \pm 0.033$ & 23.28 & $3.529 \times 10^{-05}$ \\
\hline 350 & $0.056 \pm 0.008$ & $0.245 \pm 0.069$ & $0.144 \pm 0.028$ & $0.146 \pm 0.021$ & 20.52 & 0.0001 \\
\hline 400 & $0.057 \pm 0.003$ & $0.278 \pm 0.073$ & $0.148 \pm 0.030$ & $0.157 \pm 0.043$ & 23.61 & $3.014 \times 10^{-05}$ \\
\hline 450 & $0.058 \pm 0.006$ & $0.228 \pm 0.091$ & $0.145 \pm 0.034$ & $0.175 \pm 0.043$ & 17.95 & 0.0005 \\
\hline 500 & $0.058 \pm 0.008$ & $0.259 \pm 0.054$ & $0.146 \pm 0.032$ & $0.183 \pm 0.057$ & 17.27 & 0.0006 \\
\hline 550 & $0.062 \pm 0.008$ & $0.263 \pm 0.072$ & $0.126 \pm 0.016$ & $0.180 \pm 0.052$ & 16.08 & 0.0011 \\
\hline 600 & $0.059 \pm 0.004$ & $0.271 \pm 0.036$ & $0.158 \pm 0.016$ & $0.180 \pm 0.056$ & 15.33 & 0.0016 \\
\hline 650 & $0.065 \pm 0.008$ & $0.259 \pm 0.033$ & $0.165 \pm 0.037$ & $0.184 \pm 0.045$ & 13.73 & 0.0033 \\
\hline 700 & $0.056 \pm 0.005$ & $0.261 \pm 0.073$ & $0.157 \pm 0.007$ & $0.195 \pm 0.060$ & 11.36 & 0.0099 \\
\hline
\end{tabular}

Table 3. Comparison of Poincare index $\mathrm{SD}_{12}$ ratio for different stride segment lengths at lag $=1$ in the four groups (HC, ALS, PD, and HD). All the values are expressed as mean \pm SD. Seg. Len: stride segment length, HC: healthy control, ALS: amyotrophic lateral sclerosis, PD: Parkinson’s disease, and HD: Huntington’s disease.

\begin{tabular}{ccccccc}
\hline \multirow{2}{*}{$\begin{array}{c}\text { Seg. } \\
\text { Len. }\end{array}$} & \multicolumn{4}{c}{ SD $_{12}$ ratio } & \multicolumn{2}{c}{ Kruskal-Wallis test } \\
\cline { 2 - 7 } & HC & ALS & PD & HD & Chi-sq & $p$-value \\
\hline 200 & $0.489 \pm 0.064$ & $0.643 \pm 0.178$ & $0.567 \pm 0.161$ & $0.689 \pm 0.131$ & 9.84 & 0.020 \\
250 & $0.526 \pm 0.071$ & $0.524 \pm 0.172$ & $0.630 \pm 0.151$ & $0.665 \pm 0.149$ & 5.03 & 0.169 \\
300 & $0.440 \pm 0.027$ & $0.496 \pm .0 .200$ & $0.549 \pm 0.120$ & $0.665 \pm 0.161$ & 10.74 & 0.013 \\
350 & $0.433 \pm 0.043$ & $0.477 \pm 0.127$ & $0.481 \pm 0.130$ & $0.623 \pm 0.137$ & 5.32 & 0.150 \\
400 & $0.423 \pm 0.042$ & $0.396 \pm 0.186$ & $0.408 \pm 0.097$ & $0.610 \pm 0.121$ & 5.36 & 0.148 \\
450 & $0.418 \pm 0.048$ & $0.522 \pm 0.176$ & $0.447 \pm 0.124$ & $0.628 \pm 0.141$ & 4.18 & 0.242 \\
500 & $0.407 \pm 0.034$ & $0.427 \pm 0.202$ & $0.497 \pm 0.072$ & $0.576 \pm 0.098$ & 2.91 & 0.406 \\
550 & $0.388 \pm 0.019$ & $0.506 \pm 0.227$ & $0.499 \pm 0.110$ & $0.533 \pm 0.078$ & 3.55 & 0.314 \\
600 & $0.393 \pm 0.025$ & $0.463 \pm 0.198$ & $0.420 \pm 0.077$ & $0.530 \pm 0.081$ & 2.61 & 0.456 \\
650 & $0.396 \pm 0.034$ & $0.484 \pm 0.217$ & $0.421 \pm 0.064$ & $0.522 \pm 0.094$ & 1.89 & 0.596 \\
700 & $0.429 \pm 0.027$ & $0.454 \pm 0.180$ & $0.432 \pm 0.069$ & $0.530 \pm 0.119$ & 1.18 & 0.758 \\
\hline
\end{tabular}

Table 4. Statistical significance of Poincare indices between healthy control (HC) and neurodegenerative disease groups for stride segment length = 200 strides and lag = 1 using Mann-Whitney rank sum test.

\begin{tabular}{cccc}
\hline Poincare Inde $x$ & Group 1 & Group 2 & $p$-value \\
\hline \multirow{2}{*}{$\mathrm{SD}_{1}$} & HC & ALS & $1.495 \times 10^{-04}$ \\
& HC & PD & $4.930 \times 10^{-07}$ \\
& HC & HD & $6.376 \times 10^{-08}$ \\
$\mathrm{SD}_{2}$ & HC & ALS & 0.002 \\
& HC & PD & $9.434 \times 10^{-05}$ \\
& HC & HD & $6.854 \times 10^{-06}$ \\
$\mathrm{SD}_{12}$ ratio & HC & ALS & 0.216 \\
& HC & PD & 0.176 \\
\hline
\end{tabular}




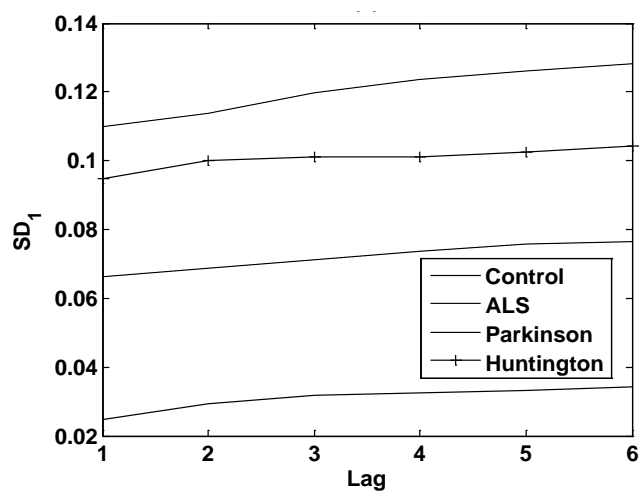

(a)

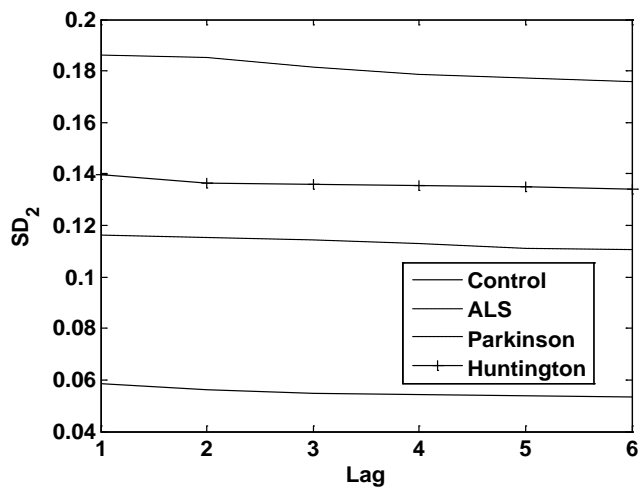

(b)

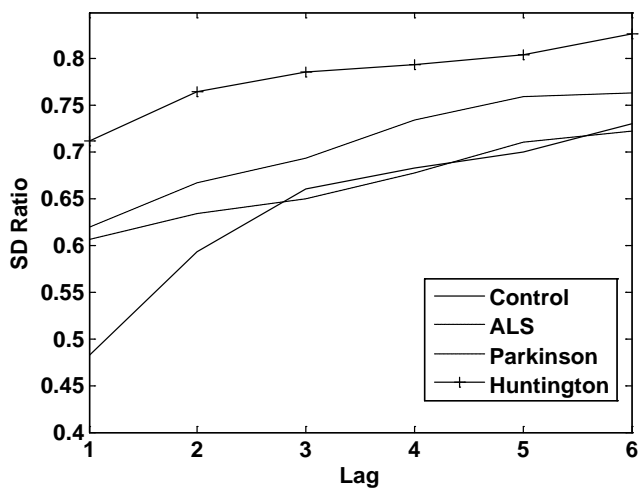

(c)

Figure 3. Lag response of Poincare plot indices in control subject, ALS patient, Huntington's patient, and Parkinson's patient for sequences of length $=200$ strides. (a) $\mathrm{SD}_{1}$, (b) $\mathrm{SD}_{2}$, and (c) $\mathrm{SD}_{1} / \mathrm{SD}_{2}$ ratio.

model-fit are tabulated in Table 5 through Table 7. The curvilinearity of lag-response of a Poincare index was evaluated using quadratic polynomial regression and the model-fit was assessed using $\mathrm{R}^{2}$ values. It is found that $\mathrm{R}^{2} \geq 0.8159$.The following points can be noted. The coefficients of quadratic terms were insignificant in all the neurodegenerative disorder groups (ALS, PD, and HD groups) and showed diminished curvilinearity, irrespective of the stride segment length. In contrast, coefficients of quadratic terms in the healthy controls were significant and exhibited curvilinearity, irrespective of the stride segment length.

The important findings of this work are summarised below. The autocovariance information contained in $m$-lagged Poincare plots can be employed to capture short-range correlations of gait variability through the behaviour of Poincare indices in health as well as disease. The analysis showed that the Poincare indices $\mathrm{SD}_{1}$ and $\mathrm{SD}_{2}$ can be readily employed to discern healthy control from neurodegenerative disorder subjects. However, $\mathrm{SD}_{12}$ ratio cannot be used directly to separate healthy from the diseased in the gait analysis. It is also found that the coefficients of quadratic terms of the $\left(\mathrm{SD}_{1}, \mathrm{SD}_{2}\right.$, and also, $\left.\mathrm{SD}_{12}\right)$ lag response in the healthy controls were significant and the lag responses exhibited curvilinearity. The coefficients of quadratic terms in all the neurodegenerative disorder groups, on the other hand, were insignificant and the lag responses showed either diminished or loss of curvilinearity.

This study has a number of limitations. 1) In general, factors like high variance, age differences, and differing male-to-female ratios between groups will have an impact on the results when statistical analyses are carried out on small sample sizes. However, it has been shown that the effect of gender on usual gait patterns is considerably small [16]. Though the effect of age on gait is complex, the effect of neurodegenerative disorders considerably predominates over the aging effects. 2) Subjects were also not perfectly matched with respect to height. However, it has been shown that the influence of height on usual gait patterns is significantly small [6]. 3) Another limitation of this study is small sample size. This is because neurodegenerative disordered subjects capable of walking independently for 5 minutes were only selected. Acquiring longer data from the same subjects is difficult as stress may interfere with the outcome of the disease. This brings a strong restriction on acquiring data. 


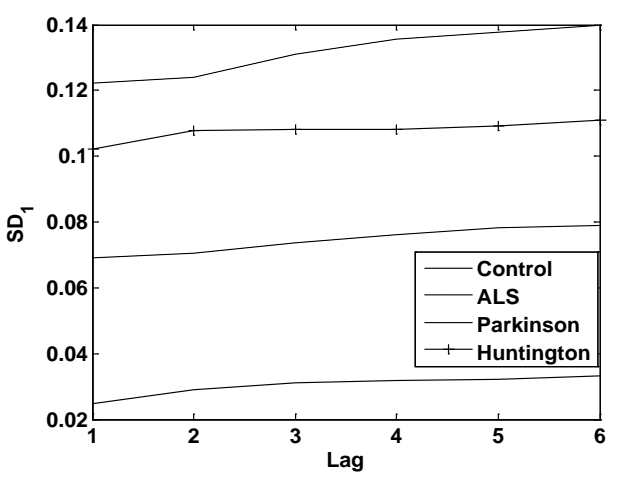

(a)

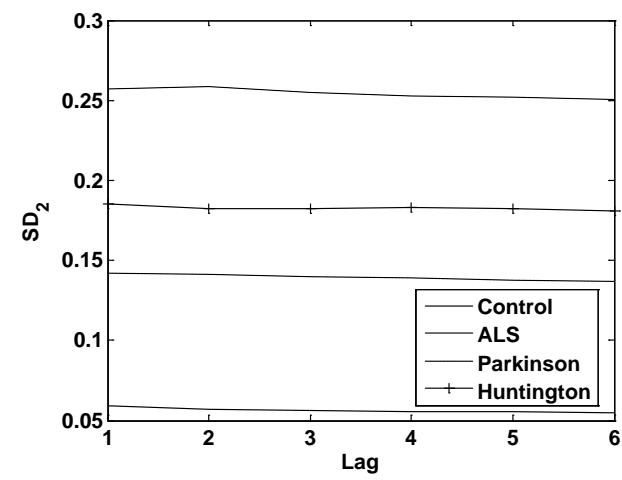

(b)

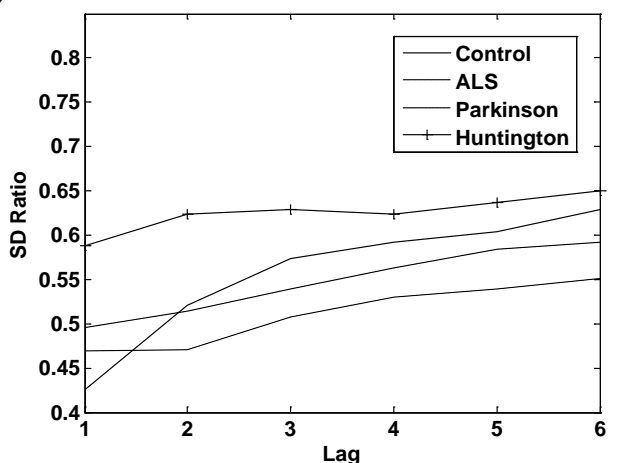

(c)

Figure 4. Lag response of Poincare plot indices in control subject, ALS patient, Huntington's patient, and Parkinson's patient for sequences of length $=500$ strides. (a) $\mathrm{SD}_{1}$, (b) $\mathrm{SD}_{2}$, and (c) $\mathrm{SD}_{1} / \mathrm{SD}_{2}$ ratio.

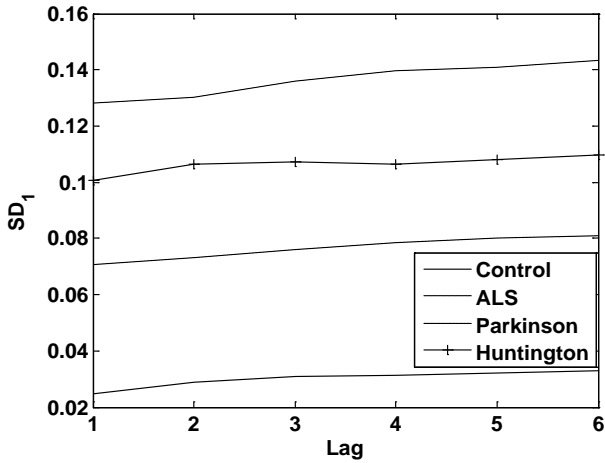

(a)

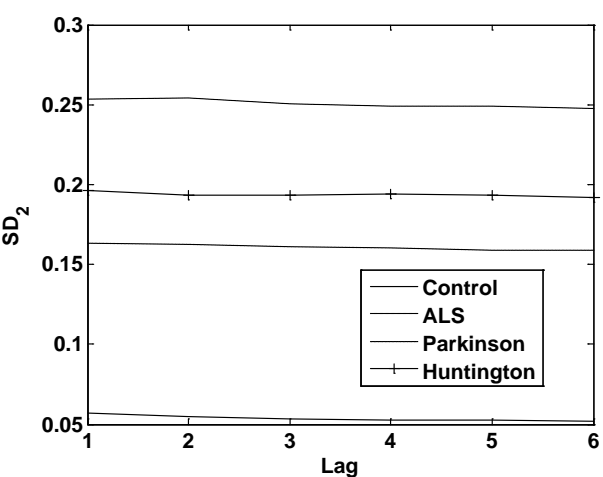

(b)

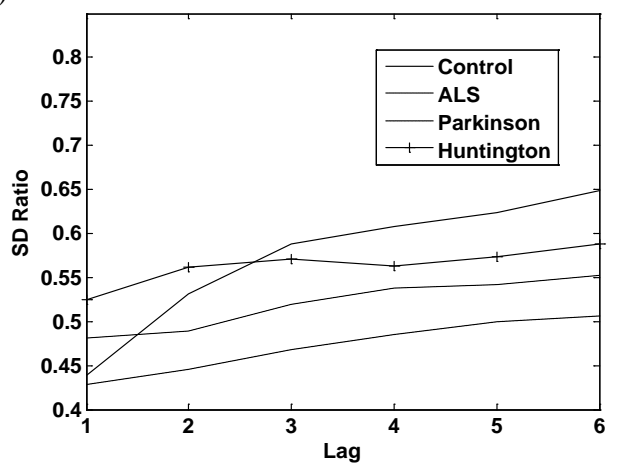

(c)

Figure 5. Lag response of Poincare plot indices in control subject, ALS patient, Huntington's patient, and Parkinson's patient for sequences of length $=700$ strides. (a) $\mathrm{SD}_{1}$, (b) $\mathrm{SD}_{2}$, and (c) $\mathrm{SD}_{1} / \mathrm{SD}_{2}$ ratio. 
Table 5. Second-order coefficients (Coeft.) and statistical significance of lag response of Poincare indices for stride segment length $=200$ strides, in the four groups (HC, ALS, PD, and HD). All the values are expressed as mean \pm SD. HC: healthy control, ALS: amyotrophic lateral sclerosis, PD: Parkinson's disease, and HD: Huntington's disease.

\begin{tabular}{|c|c|c|c|c|c|c|c|c|}
\hline \multirow{2}{*}{ Poincare Inde $\times$ - } & \multicolumn{2}{|c|}{ HC } & \multicolumn{2}{|c|}{ ALS } & \multicolumn{2}{|c|}{ PD } & \multicolumn{2}{|c|}{ HD } \\
\hline & Coeft. & $p$-value & Coeft. & $p$-value & Coeft. & $p$-value & Coeft. & $p$-value \\
\hline $\mathrm{SD}_{1}$ & $\begin{array}{c}-0.0004 \pm \\
0.0001\end{array}$ & $2.2696 \times 10^{-06}$ & $\begin{array}{l}-0.0004 \pm \\
0.0002\end{array}$ & 0.0421 & $\begin{array}{c}-1.55 \times 10^{-05} \\
\pm 0.0002\end{array}$ & 0.3902 & $\begin{array}{c}-0.0003 \pm \\
0.0004\end{array}$ & 0.2921 \\
\hline $\mathrm{SD}_{2}$ & $\begin{array}{c}0.0002 \pm \\
8.54 \times 10^{-05}\end{array}$ & $6.0030 \times 10^{-06}$ & $\begin{array}{c}0.0001 \pm \\
0.0001\end{array}$ & 0.2814 & $\begin{array}{c}-5.17 \times 10^{-05} \\
\pm 0.0001\end{array}$ & 0.9872 & $\begin{array}{l}0.0001 \pm \\
0.0003\end{array}$ & 0.4081 \\
\hline $\mathrm{SD}_{12}$ ratio & $\begin{array}{c}-0.0103 \pm \\
0.0049\end{array}$ & $1.7502 \times 10^{-05}$ & $\begin{array}{c}-0.0031 \pm \\
0.0028\end{array}$ & 0.0629 & $\begin{array}{l}0.0007 \pm \\
0.0046\end{array}$ & 0.9047 & $\begin{array}{c}-0.0034 \pm \\
0.0076\end{array}$ & 0.2348 \\
\hline
\end{tabular}

Table 6. Second-order coefficients (Coeft.) and statistical significance of lag response of Poincare indices for stride segment length $=500$ strides, in the four groups (HC, ALS, PD, and HD). All the values are expressed as mean \pm SD. HC: healthy control, ALS: amyotrophic lateral sclerosis, PD: Parkinson's disease, and HD: Huntington's disease.

\begin{tabular}{|c|c|c|c|c|c|c|c|c|}
\hline \multirow{2}{*}{ Poincare Inde $\times$} & \multicolumn{2}{|c|}{$\mathrm{HC}$} & \multicolumn{2}{|c|}{ ALS } & \multicolumn{2}{|c|}{ PD } & \multicolumn{2}{|c|}{ HD } \\
\hline & Coeft. & $p$-value & Coeft. & $p$-value & Coeft. & $p$-value & Coeft. & $p$-value \\
\hline $\mathrm{SD}_{1}$ & $\begin{array}{c}-0.0003 \pm \\
0.0001\end{array}$ & 0.0054 & $\begin{array}{c}-0.0004 \pm \\
0.0002\end{array}$ & 0.2880 & $\begin{array}{c}3.446 \times 10^{-06} \\
\pm 0.0004\end{array}$ & 0.6691 & $\begin{array}{l}-0.0001 \pm \\
0.0005\end{array}$ & 0.4834 \\
\hline $\mathrm{SD}_{2}$ & $\begin{array}{c}0.0001 \pm \\
9.69 \times 10^{-05}\end{array}$ & 0.0138 & $\begin{array}{c}9.16 \times 10^{-05} \pm \\
0.0001\end{array}$ & 0.8570 & $\begin{array}{c}-4.47 \times 10^{-05} \\
\pm 0.0002\end{array}$ & 0.7805 & $\begin{array}{c}20.34 \times 10^{-05} \\
\pm 0.0002\end{array}$ & 0.6826 \\
\hline $\mathrm{SD}_{12}$ ratio & $\begin{array}{c}-0.0084 \pm \\
0.0049\end{array}$ & 0.0154 & $\begin{array}{c}-0.0016 \pm \\
0.0012\end{array}$ & 0.5025 & $\begin{array}{c}0.0013 \pm \\
0.0029\end{array}$ & 0.7186 & $\begin{array}{c}-0.0010 \pm \\
0.0033\end{array}$ & 0.5765 \\
\hline
\end{tabular}

Table 7. Second-order coefficients (Coeft.) and statistical significance of lag response of Poincare indices for stride segment length = 700 strides in the four groups (HC, ALS, PD, and HD). All the values are expressed as mean \pm SD. HC: healthy control, ALS: amyotrophic lateral sclerosis, PD: Parkinson's disease, and HD: Huntington's disease.

\begin{tabular}{|c|c|c|c|c|c|c|c|c|}
\hline \multirow{2}{*}{ Poincare Inde $x$} & \multicolumn{2}{|c|}{$\mathrm{HC}$} & \multicolumn{2}{|c|}{ ALS } & \multicolumn{2}{|c|}{ PD } & \multicolumn{2}{|c|}{ HD } \\
\hline & Coeft. & $p$-value & Coeft. & $p$-value & Coeft. & $p$-value & Coeft. & $p$-value \\
\hline $\mathrm{SD}_{1}$ & $\begin{array}{c}-0.0003 \pm \\
0.0001\end{array}$ & 0.0034 & $\begin{array}{c}-0.0003 \pm \\
0.0004\end{array}$ & 0.4672 & $\begin{array}{l}-0.0002 \pm \\
0.0005\end{array}$ & 0.5264 & $\begin{array}{c}-0.0004 \pm \\
0.0004\end{array}$ & 0.4186 \\
\hline $\mathrm{SD}_{2}$ & $\begin{array}{c}0.0002 \pm \\
9.30 \times 10^{-05}\end{array}$ & 0.0218 & $\begin{array}{c}0.0001 \pm \\
0.0002\end{array}$ & 0.6863 & $\begin{array}{c}5.48 \times 10^{-05} \\
\pm 0.0002\end{array}$ & 0.7493 & $\begin{array}{c}0.0001 \pm \\
0.0002\end{array}$ & 0.5077 \\
\hline $\mathrm{SD}_{12}$ ratio & $\begin{array}{c}-0.0087 \pm \\
0.0041\end{array}$ & 0.0155 & $\begin{array}{c}-0.0018 \pm \\
0.0020\end{array}$ & 0.4498 & $\begin{array}{c}-0.0016 \pm \\
0.0035\end{array}$ & 0.5595 & $\begin{array}{c}-0.0021 \pm \\
0.0044\end{array}$ & 0.3949 \\
\hline
\end{tabular}

In any case, this study is a compromise between a classical single case study and a cross-sectional survey and it is possible to arrive at reliable results with a small number of participants. This implies that the discrimination using this method stands irrespective of the above limitations. Nevertheless, further research related to larger subject groups and spread across many age groups and ethnicity is recommended. Also, further understanding of the origin and mechanisms of these and other neurological disorders is essential to more completely characterize the underlying pathophysiologies.

\section{Conclusion}

This study shows that lagged gait Poincare plots have potential to provide more information by reflecting shortrange correlations of gait variability through the behaviour of Poincare indices in health as well as disease. In the context of short-term variability, a curvilinear relation between lag $(1-6)$ and Poincare indices was found in the lag response of Poincare indices in healthy subjects whereas in patients with neurodegenerative disorders this curvilinear relation was either diminished or absent depending upon the severity of the disease. 


\section{References}

[1] Winter, D.A. (2009) Biomechanics and Motor Control of Human Movement. 4th Edition, John Wiley \& Sons, Hoboken. http://dx.doi.org/10.1002/9780470549148

[2] Callisaya, M.L., Blizzard, L., McGinley, J.L., Schmidt, M.D. and Srikanth, V.K. (2010) Sensorimotor Factors Affecting Gait Variability in Older People—A Population-Based Study. Journals of Gerontology—Series A, 65, 386-392. http://dx.doi.org/10.1093/gerona/glp184

[3] Hausdorff, J.M., Peng, C.K., Ladin, Z., Wei, J.Y. and Goldberger, A.L. (1995) Is Walking a Random Walk? Evidence for Long-Range Correlations in Stride Interval of Human Gait. Journal of Applied Physiology, 78, 349-358.

[4] Bollens, B., Crevecoeur, F., Nguyen, V., Detrembleur, C. and Lejeune, T. (2010) Does Human Gait Exhibit Comparable and Reproducible Long-Range Autocorrelations on Level Ground and on Treadmill? Gait and Posture, 32, 369373. http://dx.doi.org/10.1016/j.gaitpost.2010.06.011

[5] Hausdorff, J.M., Mitchell, S.L., Firtion, R., Peng, C.K., Cudkowicz, M.E., Wei, J.Y. and Goldberger, A.L. (1997) Altered Fractal Dynamics of Gait: Reduced Stride-Interval Correlations with Aging and Huntington's Disease. Journal of Applied Physiology, 82, 262-269.

[6] Hausdorff, J.M., Lertratanakul, A., Cudkowicz, M.E., Peterson, A.L., Kaliton, D. and Goldberger, A.L. (2000) Dynamic Markers of Altered Gait Rhythm in Amyotrophic Lateral Sclerosis. Journal of Applied Physiology, 88, 20452053.

[7] Hausdorff, J.M., Cudkowicz, M.E., Firtion, R., Wei, J.Y. and Goldberger, A.L. (1998) Gait Variability and Basal Ganglia Disorders: Stride-to-Stride Variations in Gait Cycle Timing in Parkinson and Huntington's Disease. Movement Disorders, 13, 428-437. http://dx.doi.org/10.1002/mds.870130310

[8] Moody, G.B., Mark, R.G. and Goldberger, A.L. (2001) Physio Net: A Web-Based Resource, for the Study of Physiologic Signals. IEEE Engineering in Medicine and Biology Magazine, 20, 70-75. http://dx.doi.org/10.1109/51.932728

[9] Hahn, G.J. and Shapiro, S.S. (1994) Statistical Models in Engineering. Wiley, Hoboken.

[10] Brennan, M., Palaniswami, M. and Kamen, P. (2001) Do Existing Measures of Poincare Plot Geometry Reflect Nonlinear Features of Heart Rate Variability? IEEE Transactions on Biomedical Engineering, 48, 1342-1347. http://dx.doi.org/10.1109/10.959330

[11] Karmakar, C., Khandoker, A., Gubbi, J. and Palaniswami, M. (2009) Complex Correlation Measure: A Novel Descriptor for Poincare Plot. BioMedical Engineering, 8, 17. http://dx.doi.org/10.1186/1475-925x-8-17

[12] Goshvarpour, A., Goshvarpour, A. and Rahati, S. (2011) Analysis of Lagged Poincare Plots in Heart Rate Signals during Meditation. Digital Signal Processing, 21, 208-214. http://dx.doi.org/10.1016/j.dsp.2010.06.015

[13] Stein, P.K. and Reddy, A. (2005) Non-Linear Heart Rate Variability and Risk Stratification in Cardiovascular Disease. Indian Pacing and Electrophysiology Journal, 5, 210-220.

[14] Lerma, C., Infante, O., Perez-Grovas, H. and Jose, M.V. (2003) Poincare Plot Indexes of Heart Rate Variability Capture Dynamic Adaptations after Haemodialysis in Chronic Renal Failure Patients. Clinical Physiology and Functional Imaging, 23, 72-80. http://dx.doi.org/10.1046/j.1475-097X.2003.00466.X

[15] Thakre, T.P. and Smith, M.L. (2006) Loss of Lag-Response Curvilinearity of Indices of Heart Rate Variability in Congestive Heart Failure. BMC Cardiovascular Disorders, 6, 1-10. http://dx.doi.org/10.1186/1471-2261-6-27

[16] Gabell, A. and Nayak, U.S.L. (1984) The Effect of Age on Variability in Gait. Journal of Gerontology, 39, 662-666. http://dx.doi.org/10.1093/geronj/39.6.662 